\title{
Ultrasound and microbubble mediated drug delivery: acoustic pressure as determinant for uptake via membrane pores or endocytosis
}

Ine De Cock ${ }^{1}$, Elisa Zagato ${ }^{1}$, Kevin Braeckmans ${ }^{1}$, Ying Luan ${ }^{2}$, Nico de Jong ${ }^{2}$, Stefaan C. De Smedt $^{1^{*}}$, Ine Lentacker ${ }^{1}$

${ }^{1}$ Laboratory of General Biochemistry and Physical Pharmacy, Ghent Research Group on Nanomedicine, Faculty of Pharmaceutical Sciences, Ghent University, Ottergemsesteenweg 460, Ghent, Belgium

${ }^{2}$ Biomedical Engineering Thoraxcenter, Erasmus Medical Center, P.O. Box 2040, 3000 CA, Rotterdam, The Netherlands

* Corresponding author at: Laboratory of General Biochemistry and Physical Pharmacy, Ottergemsesteenweg 460, 9000 Ghent, Belgium. Tel.:+32 926480 78; fax: +32 92648189. E-mail address: Stefaan.DeSmedt@UGent.be (S.C. De Smedt).

Corresponding author during submission procedure: Ine De Cock, Laboratory of General Biochemistry and Physical Pharmacy, Ottergemsesteenweg 460, 9000 Ghent, Belgium. Tel.: +32 926480 74. E-mail address: Ine.DeCock@UGent.be

\begin{abstract}
Although promising results are achieved in ultrasound mediated drug delivery, its underlying biophysical mechanisms remain to be elucidated. Pore formation as well as endocytosis has been reported during ultrasound application. Due to the plethora of ultrasound settings used in literature, it is extremely difficult to draw conclusions on which mechanism is actually involved. To our knowledge, we are the first to show that acoustic pressure influences which route of drug uptake is addressed, by inducing different microbubble-cell interactions. To investigate this, FITC-dextrans were used as model drugs and their uptake was analyzed by flow cytometry. In fluorescence intensity plots, two subpopulations arose in cells with FITCdextran uptake after ultrasound application, corresponding to cells having either low or high uptake. Following separation of the subpopulations by FACS sorting, confocal images indicated that the low uptake population showed endocytic uptake. The high uptake population represented uptake via pores. Moreover, the distribution of the subpopulations shifted to the high uptake population with increasing acoustic pressure. Real-time confocal recordings during ultrasound revealed that membrane deformation by microbubbles may be the trigger for endocytosis via mechanostimulation of the cytoskeleton. Pore formation was shown to be caused by microbubbles propelled towards the cell. These results provide a better insight in the role of acoustic pressure in microbubble-cell interactions and the possible consequences for drug uptake. In addition, it pinpoints the need of a more rational, microbubble behavior based choice of acoustic parameters in ultrasound mediated drug delivery experiments.
\end{abstract}

\section{Keywords}

Ultrasound, microbubbles, acoustic pressure, sonoporation, endocytosis, drug delivery 


\section{INTRODUCTION}

While ultrasound is a well-established diagnostic imaging technique, its great potential in enhancing drug delivery was only acknowledged in the late 1990s [1]. Since then, the ultrasound mediated uptake of small drugs [2], proteins [3], as well as larger nanoparticles such as gene complexes [4] or drug loaded liposomes [5] has been reported. In these studies, ultrasound is often used in combination with microbubbles. These are micrometer sized (1-10 $\mu \mathrm{m})$ structures with a gas core stabilized by a lipid, polymer or protein shell. Due to their compressible core, they can cavitate in an ultrasonic field. Cavitation is the alternating shrinking and expanding of the microbubbles, according to the pressure phases of the ultrasound wave [6]. Microbubbles were originally developed as contrast agents for ultrasound imaging. Cavitation produces backscatter of the ultrasound waves, thereby intensifying the reflected signal [7]. However, microbubbles are also essential for drug delivery purposes, because they amplify the biophysical effects of ultrasound. Depending on the ultrasound intensities used, two major types of microbubble cavitation are observed: stable and inertial cavitation. During stable cavitation, microbubbles show continuous lowamplitude oscillations, generating microstreamings in the surrounding fluid. When using higher ultrasound intensities, inertial cavitation occurs with larger oscillations and eventually microbubble implosion. This is accompanied by more violent phenomena, such as microjets and shock waves [8].

Whereas microbubble cavitation in an ultrasonic field has been studied extensively, the biophysical mechanisms leading to enhanced drug delivery are still a matter of debate. The formation of membrane pores, i.e. sonoporation, is generally accepted as main mechanism by which drugs enter cells during ultrasound application. Scanning electron microscopy images showed clear membrane disruptions after ultrasound exposure [9]. By voltage clamp techniques, an increase in transmembrane current simultaneously with ultrasound application was reported, indicating ion flux through pores [10]. Moreover, several papers demonstrated the uptake of cell-impermeable molecules when exposing cells to ultrasound [11-13]. However, more recently, Meijering et al. [14] stated that besides pore formation, enhanced endocytosis also contributes to ultrasound mediated delivery. Molecule size was observed to determine decisively which route of uptake occurs, with endocytosis playing a greater role for larger molecules. A few other studies confirmed that endocytosis is indeed involved in ultrasound mediated uptake [15-18]. In contrast, Schlicher et al. [19] blocked endocytic routes and found no difference in uptake of calcein. Following Meijering's hypothesis, this might be explained by the small size of calcein. However, our group found that the uptake of large gene complexes loaded on microbubbles was not affected by endocytosis inhibitors [20]. Hence, the complexes were directly delivered in the cytoplasm via pores. We hypothesize that this discrepancy in literature might be due to the large variety of ultrasound settings used. It is striking that papers arguing in favour of endocytosis mostly used modest ultrasound conditions. In contrast, papers proving that endocytosis is not involved mostly used higher ultrasound intensity regimes.

Therefore, we investigated in the first part of this study if the route of uptake, via pores or via endocytosis, is dependent on the acoustic pressure used. Moreover, the uptake of low and high molecular weight dextrans was compared to evaluate the role of molecule size. In the second part, we performed real-time confocal microscopy during ultrasound radiation to reveal microbubble-cell interactions. The swept field confocal technology we implemented allows imaging at a relatively high frame rate of 15 fps (frames per second), while maintaining confocal resolution. In this way, our aim was to link microbubble-cell interactions to mechanisms involved in drug uptake. 


\section{MATERIALS AND METHODS}

\subsection{Cell culture}

Human melanoma cells (BLM cells) [21], were grown in culture flasks in a humidified atmosphere with $5 \% \mathrm{CO}_{2}$ at $37^{\circ} \mathrm{C}$. The culture medium was Dulbecco's Modified Eagle Medium with Nutrient Mixture F12 (Gibco, Merelbeke, Belgium), supplemented with 10\% (v/v) fetal bovine serum (FBS) (Hyclone, Thermo Scientific, MA, USA), $20 \mathrm{U} / \mathrm{ml}$ penicillinstreptomycin (Gibco, Merelbeke, Belgium), 2 mM L-glutamine (Gibco, Merelbeke, Belgium) and $10 \mathrm{mM}$ HEPES (4-(2-hydroxyethyl)-1-piperazineethanesulfonic acid) (Sigma-Aldrich, Diegem, Belgium). One day before the experiment, cells were harvested by 0,05\% trypsinEDTA (ethylenediaminetetraacetic acid) (Gibco, Merelbeke, Belgium) and replated in Opticells $^{\text {TM }}$ (Nunc, Thermo Scientific, MA, USA). For flow cytometry experiments, cells were seeded at a density of $2 \times 10^{6}$ cells $/ \mathrm{ml}$, reaching confluency after 1 day. For microscopy experiments, a cell density of $1,3 \times 10^{6}$ cells/mL was used to obtain a sub-confluent monolayer.

\subsection{Microbubbles}

Microbubbles composed of DPPC (1,2-dipalmitoyl-sn-glycero-3-phosphocholine) (Lipoid, Ludwigshafen, Germany) and DSPE-PEG (1,2-distearoyl-sn-glycero-3phosphoethanolamine-N-[methoxy(polyethylene glycol)-2000] ammonium salt) (Avanti Polar Lipids Inc, Alabaster, AL, USA) in a 85:15 molar ratio were prepared as described previously [4]. The size and the concentration of the microbubbles in the dispersion were determined with a Multisizer ${ }^{\mathrm{TM}} 4$ (Beckman Coulter, Brea, CA, USA). Microbubbles with a mean diameter of $1.408 \mu \mathrm{m}$ and a concentration of $1.775 \times 10^{9}$ bubbles $/ \mathrm{mL}$ were obtained.

\subsection{Ultrasound equipment}

Ultrasound pulses were generated by an arbitrary waveform generator (33220A, Agilent Technologies, Diegem, Belgium) and amplified by an amplifier (150A100B, Amplifier Research Benelux, Hazerswoude Dorp, The Netherlands). The amplified electrical signals were sent to an unfocused, single element, $1 \mathrm{MHz}$ center frequency transducer (A303S-SU, Olympus Industrial Benelux, Aartselaar, Belgium), which transmits the ultrasound waves. The transducer was mounted at the side of a water tank in a $45^{\circ}$ angle and at $12 \mathrm{~cm}$ distance of the cell monolayer in the Opticell ${ }^{\mathrm{TM}}$. The water in the tank was degassed and kept at $37^{\circ} \mathrm{C}$. The Opticell ${ }^{\mathrm{TM}}$ chamber was marked with 9 exposure areas of $7 \mathrm{~mm}$ diameter. These areas correspond to regions homogeneously exposed to ultrasound, as calibrated with a needle hydrophone. The transmitted ultrasound signals were monitored with an oscilloscope (TDS 210, Tektronix, Bracknell, UK). A schematic representation of the setup is depicted in Figure 1. As described below, the ultrasound setup was mounted on a swept field confocal microscope for real-time confocal recordings.

\subsection{Uptake of FITC-dextran at different acoustic pressures}

FITC (fluorescein isothiocyanate)-dextrans $(0.25 \mathrm{mg} / \mathrm{mL}$, excitation/emission maxima: 490/520 nm, Sigma-Aldrich, Diegem, Belgium) and microbubbles $\left(8.875 \times 10^{6}\right.$ bubbles $\left./ \mathrm{mL}\right)$ were added to the cells in Opti-MEM ${ }^{\circledR}$ (Gibco, Merelbeke, Belgium). Dextrans of two molecular weights were tested, i.e. $4 \mathrm{kDa}$ and 2 MDa FITC-dextran with an estimated radius of $1.23 \mathrm{~nm}$ and $32.78 \mathrm{~nm}$ [22], respectively. The Opticell ${ }^{\mathrm{TM}}$ was flipped to allow the microbubbles to rise against the cell monolayer and submerged in the water tank. Subsequently, each exposure area marked on the Opticell ${ }^{\mathrm{TM}}$ was radiated with ultrasound pulses with a center frequency of $1 \mathrm{MHz}$, a pulse length of 2000 cycles and a repetition rate of 
$125 \mathrm{~Hz}$. The total duration of sonication was 5 sec for each exposure area. The acoustic pressure ranged from 100 to $500 \mathrm{kPa}$ between Opticells ${ }^{\mathrm{TM}}$. Following ultrasound exposure, cells were placed in the incubator until a total incubation time of 15 min with FITC-dextran was completed. Afterwards, exposure areas were cut out from the Opticell ${ }^{\mathrm{TM}}$ and transferred to a well plate. Cells were collected by trypsinization and cells of three exposure areas were pooled. Before flow cytometric analysis Calcein AM Red ${ }^{\mathrm{TM}}$ viability staining (excitation/emission maxima: 647/659 nm, Assay Biotech, Sunnyvale, CA, USA) was performed. Flow cytometric data were acquired using a FACSCalibur ${ }^{\mathrm{TM}}$ (BD, Erembodegem, Belgium) and analyzed using CellQuestPro ${ }^{\mathrm{TM}}$ software. Experiments were performed in threefold.

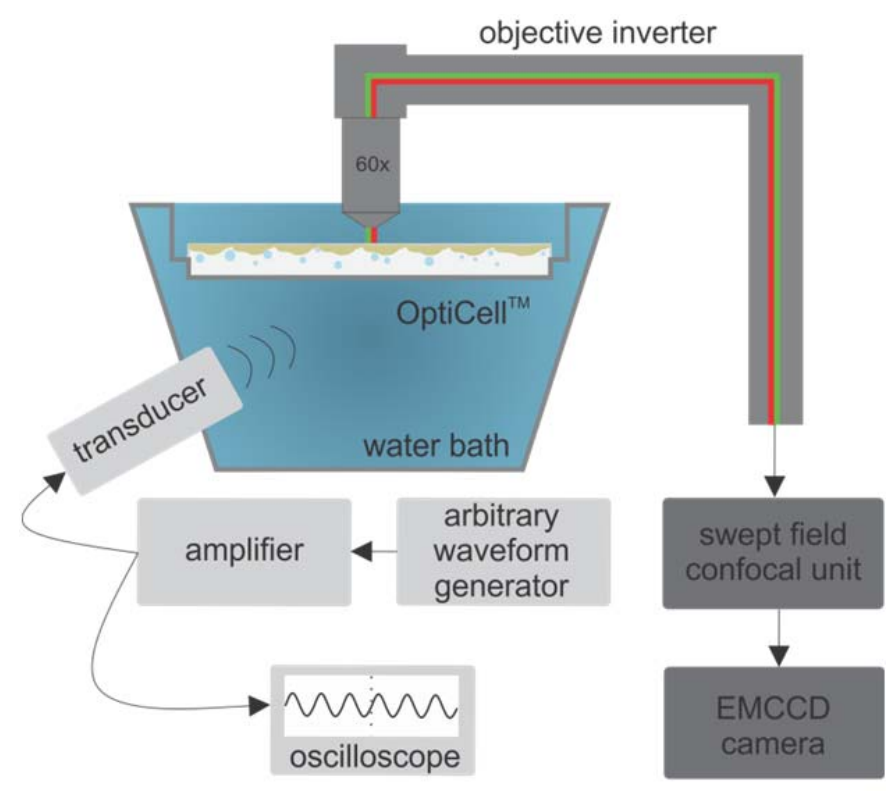

Figure 1. Schematic representation of the ultrasound and swept field confocal microscope setup. The Opticell $^{\mathrm{TM}}$ containing cells and microbubbles was submerged in a water bath. The ultrasound transducer was positioned below the Opticell ${ }^{\mathrm{TM}}$, while imaging occurred through a $60 \mathrm{x}$ water dipping lens placed on top by an objective inverter. Images were acquired by a swept field confocal unit and an EMCCD camera, operating at a frame rate of $15 \mathrm{fps}$.

\subsection{FACS sorting and intracellular localization of FITC-dextran}

Incubation with 2 MDa FITC-dextran and exposure to ultrasound was performed according to the same protocol as described in section 2.4. Following trypsinization, cells of all 9 exposure areas were pooled, resuspended in PBS (phosphate buffered saline) (Gibco, Merelbeke, Belgium) supplemented with 1\% FBS (Hyclone, Thermo Scientific, MA, USA) and $2 \mathrm{mM}$ Titriplex ${ }^{\circledR}$ III (ethylenedinitrilotetraacetic acid disodium salt dehydrate) (Merck KGaA, Darmstadt, Germany), and filtered through a cell strainer (mesh $35 \mu \mathrm{m}$ ). Based on FITCintensity, two subpopulations were sorted by a FACS Aria ${ }^{\mathrm{TM}}$ III flow cytometer (BD, Erembodegem, Belgium). Subsequently, the intracellular localization of FITC-dextran of the subpopulations was evaluated by confocal microscopy. Co-localization of FITC-dextran with lysosomes was assessed by staining the lysosomes with $400 \mathrm{nM}$ LysoTracker ${ }^{\circledR}$ Red DND-99 (excitation/emission maxima: 577/590, Molecular Probes, Gent, Belgium) for $30 \mathrm{~min}$. Images were acquired by a Nikon C1si confocal laser scanning module attached to a motorized TE2000-E inverted microscope (Nikon Benelux, Brussels, Belgium), using a Plan Apo VC 60x 1.4 NA oil immersion lens (Nikon). For the co-localization experiment, sequential acquisition of FITC-dextran and LysoTracker® Red DND-99 was performed to avoid bleedthrough of the two fluorescent dyes. 


\subsection{Real-time confocal microscopy during ultrasound exposure}

The cell membrane of BLM cells was labeled with $4 \mu \mathrm{g} / \mathrm{mL}$ CellMask ${ }^{\mathrm{TM}}$ Deep Red Plasma membrane Stain (excitation/emission maxima: 649/666 nm, Molecular Probes, Gent, Belgium). Fluorescent microbubbles, with $0.5 \mathrm{~mol} \%$ of the fluorescent lipid cholesterylBODIPY ${ }^{\circledR}$ FL $\mathrm{C}_{12}$ (excitation/emission maxima: 500/510 nm, Molecular probes, Gent, Belgium) incorporated in the lipid shell, were prepared similar to non-fluorescent microbubbles. They were added to cells in a concentration of $4.438 \times 10^{6} / \mathrm{mL}$. In addition, the medium was supplemented with $25 \mu \mathrm{g} / \mathrm{mL}$ propidium iodide (PI) (excitation/emission maxima: 535/617 nm, Enzo Life Sciences, Antwerpen, Belgium) as marker for pore formation, since PI is excluded from cells with intact cell membranes. Cells were exposed to ultrasound pulses with a center frequency of $1 \mathrm{MHz}$, a pulse length of 2000 cycles and a repetition rate of $125 \mathrm{~Hz}$ for a total duration of $30 \mathrm{sec}$. The acoustic pressure was varied from 100 to $500 \mathrm{kPa}$. To record microbubble-cell interactions in real time, the ultrasound setup was mounted on an Eclipse Ti inverted microscope (Nikon), equipped with an objective inverter (LSM TECH, Wellsville, PA, USA) and an NIR Apo 60x 1.0 NA water dipping lens (Nikon) (Figure 1). The microscope was connected to a LiveScan ${ }^{\mathrm{TM}}$ swept field confocal unit (Nikon), allowing relatively fast imaging (frame rate of $15 \mathrm{fps}$ ) while maintaining confocal resolution. This confocal unit was equipped with a 488/647 nm dichroic mirror and a 505/585/685 nm Brightline ${ }^{\circledR}$ triple-band bandpass filter (Semrock, Rochester, NY, USA). Fluorophores were excited by a $488 \mathrm{~nm}$ and $640 \mathrm{~nm}$ laser (Monolithic Laser Combiner MLC 400B, Agilent Technologies, Diegem, Belgium). Images were acquired with the NIS Elements AR software using an EMCCD camera (iXon Ultra 897, Andor Technology, CT, USA), starting from 5 sec before the onset of ultrasound exposure and for a total duration of $1 \mathrm{~min}$. Recordings were analyzed with Image $\mathrm{J}$.

\section{RESULTS}

\subsection{Uptake of FITC-dextran at different acoustic pressures}

The influence of the acoustic pressure on the uptake of $4 \mathrm{kDa}$ and $2 \mathrm{MDa}$ FITC-dextran was evaluated by flow cytometry. In these experiments, cells were exposed to microbubbles and 4 $\mathrm{kDa}$ or $2 \mathrm{MDa}$ FITC-dextrans. Subsequently, they were treated with ultrasound of different acoustic pressures, ranging from $100 \mathrm{kPa}$ to $500 \mathrm{kPa}$. As a control sample, cells with microbubbles were sonicated in the absence of dextrans (US - FD sample) to verify that ultrasound treatment did not augment autofluorescence. Following ultrasound exposure, cells were analyzed with flow cytometry. Moreover, cell viability was assessed with the Calcein AM Red ${ }^{\mathrm{TM}}$ viability stain. All gated cells were found to be viable. However, a higher percentage of cell debris was detected with increasing acoustic pressure, up to $23 \%$ at 500 $\mathrm{kPa}$. Consequently, increasing the acoustic pressure led to a higher toxicity. Examples of fluorescence intensity plots indicating the uptake of 2MDa dextrans are presented in Supplementary Figure 1. Similar plots were obtained for 4 kDa FITC-dextran. In these plots, both for $2 \mathrm{MDa}$ and $4 \mathrm{kDa}$ dextrans, two subpopulations could be distinguished, corresponding to cells with either low or high uptake of dextrans. In Figure 2, these data are expressed as the percentage of cells (A-C) showing either low uptake of FITC-dextrans (low intensity population - LIP) or high uptake (high intensity population - HIP) and the corresponding mean fluorescence intensity (MFI, B-D). 

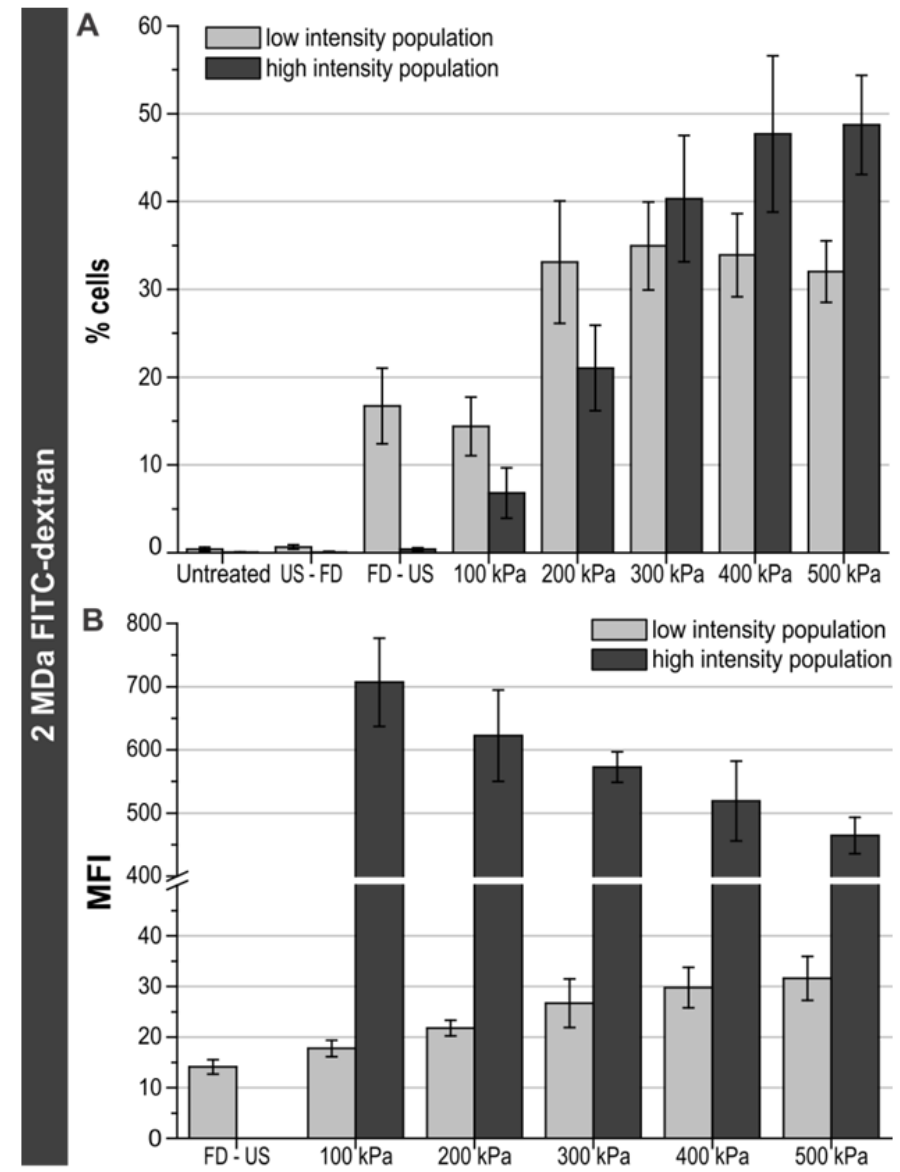

Figure 2. Uptake of 2 MDa FITCdextran (A and $\mathrm{B}$ ) and $4 \mathrm{kDa}$ FITCdextran (C and $D)$. Light gray bars represent the low intensity population; dark gray bars correspond to the high intensity population. For each subpopulation, the percentage positive cells (A and $\mathrm{C}$ ) and mean fluorescence intensity (MFI, B and D) are depicted. Results are the mean \pm standard deviation $(\mathrm{N}=3)$. The MFI of the LIP for the untreated and US-FD sample is not presented in the graph, since the number B 8007 low intensity population of cells in this LIP gate is negligible. This equally applies for the HIP MFI of the FD-US sample.

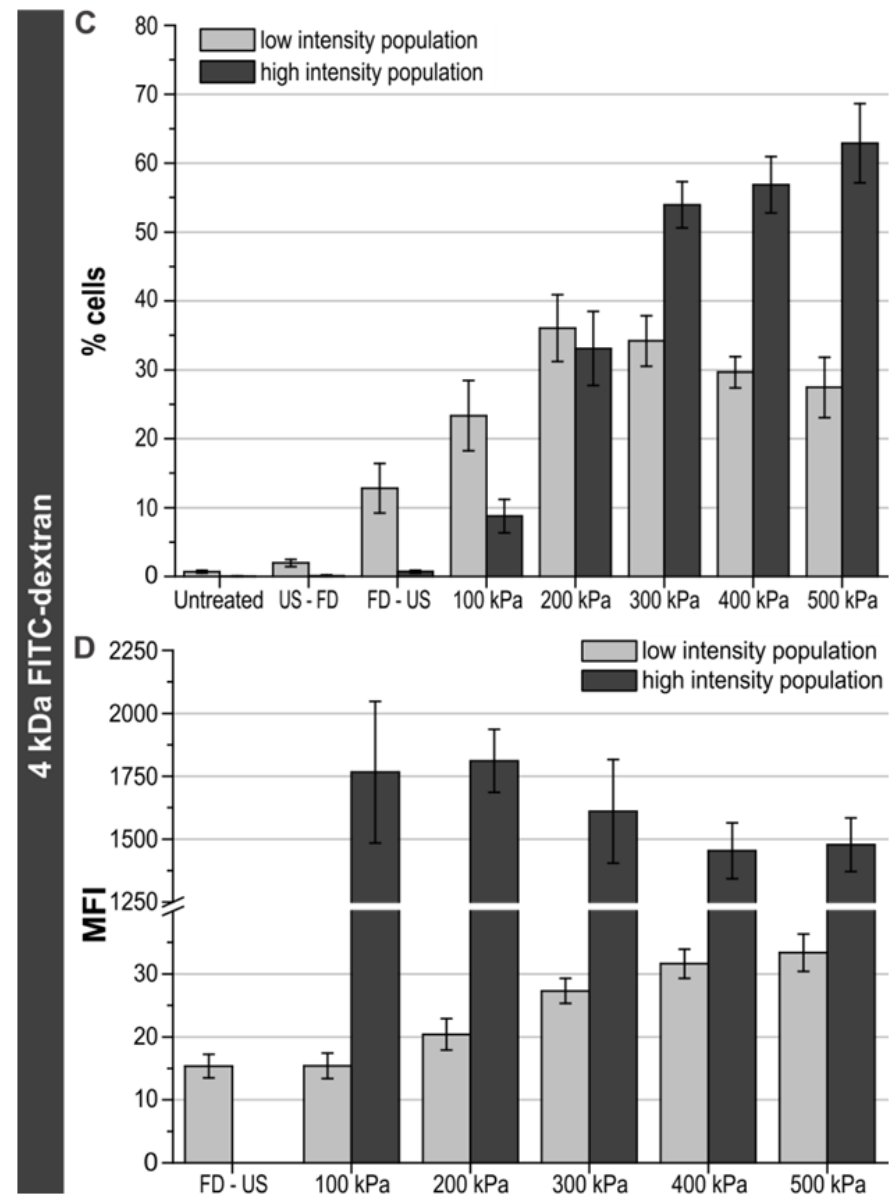


In experiments with 2 MDa FITC-dextran, when cells were incubated with dextran without ultrasound exposure (FD - US sample), there was an increase in uptake compared to control samples. This was expected, since dextrans are well endocytosed [23]. Therefore, this sample corresponded to the basal endocytic uptake. However, when cells were treated with ultrasound, an enhanced uptake in the LIP was observed. Starting from $200 \mathrm{kPa}$, the percentage positive cells reached up to $35 \%$, compared to only $17 \%$ for the basal endocytic uptake. The MFI of the LIP increased with increasing acoustic pressure. Moreover, for sonicated cells, a second subpopulation with high uptake appeared (HIP). The fraction of cells in this HIP increased from $7 \%$ at $100 \mathrm{kPa}$ to almost $50 \%$ at $500 \mathrm{kPa}$. The MFI showed a decreasing trend when higher pressures were applied.

Regarding $4 \mathrm{kDa}$ FITC-dextran, similar uptake profiles and trends were obtained. The percentage positive cells as well as the MFI of the LIP increased when exposing cells to ultrasound, reaching similar maximum values (34\% positive cells and an MFI of 31) as for 2 MDa FITC-dextran (35\% positive cells and an MFI of 32). This maximum percentage decreased at higher pressures for $4 \mathrm{kDa}$ dextrans. This drop may be attributed to the high number of positive cells in the HIP (63\%). Since a total percentage positive cells of almost $100 \%$ is reached when summing up the LIP and HIP, the increase in HIP must be compensated by a decrease in LIP. Furthermore, the maximum percentage positive cells as well as maximum MFI values of the HIP were substantially higher for $4 \mathrm{kDa}$ compared to 2 MDa FITC-dextran.

\subsection{FACS sorting and intracellular localization of FITC-dextran}

Since the basal endocytic uptake only led to a low intensity population, we hypothesized that the high intensity population represented uptake via another route, possibly membrane pores. To investigate this hypothesis, we analyzed the fluorescent uptake pattern of the two subpopulations by confocal microscopy. In order to separate cells of both populations, cells were sorted based on their fluorescence intensity by FACS sorting. Four samples were subjected to cell sorting, i.e. the basal uptake sample and the samples exposed to 100, 300 and $500 \mathrm{kPa}$. Microscopy images revealed three different fluorescent patterns: (i) punctuate, (ii) diffuse and (iii) mixed, having both punctuate and diffuse features (Figure 3). These patterns
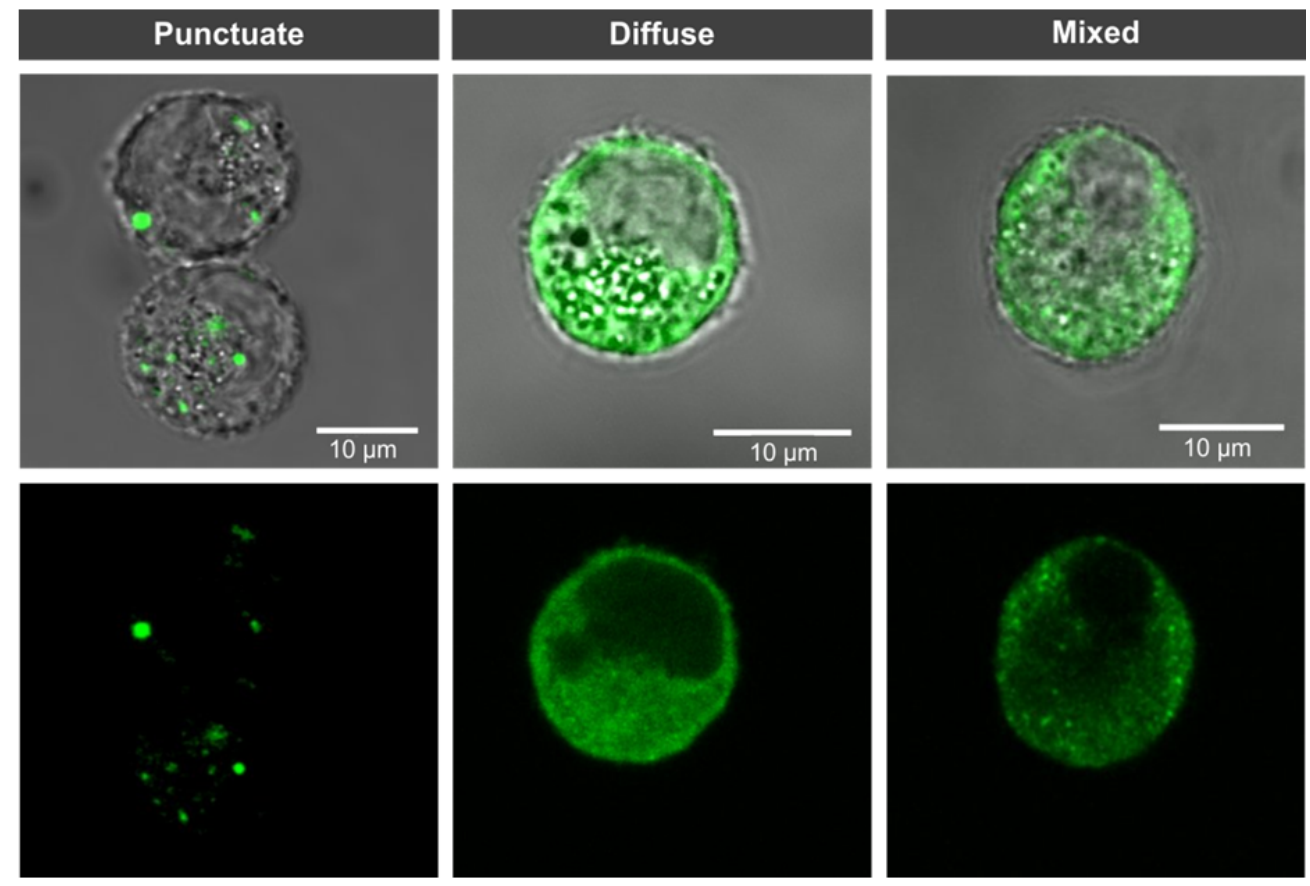
Figure 3. Intracellular localization of FITC-dextran. Confocal microscopy images with the top rows depicting an overlay of the transmission and FITC-fluorescence image; the bottom rows only show FITC-fluorescence. Three different fluorescent patterns were observed: punctuate, diffuse and mixed.

were observed for both $2 \mathrm{MDa}$ (Figure 3) and 4 kDa FITC-dextran (Supplementary Figure 2). They only differed in the presence of $4 \mathrm{kDa}$ dextrans in the nucleus in the diffuse pattern. 4 $\mathrm{kDa}$ dextrans are small enough to be transported across the nuclear membrane, while large molecules are excluded. Table 1 outlines the occurrence of the fluorescent patterns among the subpopulations. Since the patterns are clearly discernible, this was determined by visual analysis. The LIP of the basal endocytic uptake sample showed a punctuate fluorescent pattern, as expected, since the FITC-dextrans are pocketed in endocytic vesicles. Remarkably, the vast majority of the cells in the LIP of radiated samples had this speckled pattern as well. This proved that the LIP of ultrasound exposed cells showed endocytic uptake. In contrast, cells of the HIP mostly had a diffuse pattern, suggesting uptake via pores. Pores allow passive diffusion of the FITC-dextrans inwards the cell, after which they spread throughout the cytoplasm. Besides a diffuse fluorescent signal, a notable number of cells of the HIP had a punctuate or mixed pattern. This fraction may be attributed to errors in the cell sorting process. To determine the accuracy of cell sorting, the sorted LIP and HIP cells were reanalyzed by flow cytometry. The accuracy was defined as the percentage of cells having a fluorescence intensity within the previously set LIP or HIP gate, respectively. As described in Table 1, the accuracy for the HIP sorting was $70 \%$ and $82 \%$ for the $100 \mathrm{kPa}$ and $500 \mathrm{kPa}$ sample, respectively. This corresponded to the fraction of cells having a diffuse pattern, i.e. $72 \%$ and $80 \%$ respectively. In contrast, the accuracy for the LIP was over $90 \%$, in line with the occurrence of the punctuate pattern in the LIP. Consequently, cells having a pattern deviating from the majority may correspond to incorrect sorted cells. Therefore, it may be concluded that the LIP represents endocytic uptake, while the HIP corresponds to uptake via pores. The mixed pattern, which is not occurring frequently, may be the result of uptake via both routes.

Table 1. Fluorescent patterns in the low and high intensity subpopulation.

\begin{tabular}{|c|c|c|c|c|}
\hline & \multicolumn{4}{|c|}{ LOW INTENSITY POPULATION (LIP) } \\
\hline & $\begin{array}{c}\mathbf{F D}-\mathbf{U S} \\
\mathrm{N}=90^{\mathrm{C}}\end{array}$ & $\begin{array}{c}100 \mathbf{~ k P a} \\
\mathrm{N}=92^{\mathrm{C}}\end{array}$ & $\begin{array}{c}300 \mathbf{~ k P a} \\
\mathrm{N}=65^{\mathrm{c}}\end{array}$ & $\begin{array}{c}500 \mathbf{~ k P a} \\
\mathrm{N}=69^{\mathrm{c}}\end{array}$ \\
\hline $\begin{array}{l}\text { Punctuate }^{\mathrm{a}} \\
\text { Diffuse }^{\mathrm{a}} \\
\text { Mixed }^{\mathrm{a}}\end{array}$ & $\begin{array}{c}95,6 \% \\
1,1 \% \\
3,3 \%\end{array}$ & $\begin{array}{c}96,7 \% \\
2,2 \% \\
1,1 \%\end{array}$ & $\begin{array}{c}90,8 \% \\
4,6 \% \\
4,6 \%\end{array}$ & $\begin{array}{c}92,8 \% \\
5,8 \% \\
1,4 \%\end{array}$ \\
\hline \multirow[t]{3}{*}{ Accuracy $^{b}$} & & $96,0 \%$ & & $94,1 \%$ \\
\hline & \multicolumn{4}{|c|}{ HIGH INTENSITY POPULATION (HIP) } \\
\hline & & $\begin{array}{c}100 \mathbf{~ k P a} \\
\mathrm{N}=65^{\mathrm{c}}\end{array}$ & $\begin{array}{c}300 \mathbf{~ k P a} \\
\mathrm{N}=77^{\mathrm{C}}\end{array}$ & $\begin{array}{c}500 \mathbf{~ k P a} \\
\mathrm{N}=76^{\mathrm{c}}\end{array}$ \\
\hline $\begin{array}{l}\text { Punctuate }^{\mathrm{a}} \\
\text { Diffuse }^{\mathrm{a}} \\
\text { Mixed }^{\mathrm{a}}\end{array}$ & & $\begin{array}{l}16,9 \% \\
72,3 \% \\
10,8 \% \\
\end{array}$ & $\begin{array}{c}3,9 \% \\
81,8 \% \\
14,3 \%\end{array}$ & $\begin{array}{c}5,3 \% \\
80,3 \% \\
14,4 \%\end{array}$ \\
\hline Accuracy $^{\mathbf{b}}$ & & $70,2 \%$ & & $81,8 \%$ \\
\hline
\end{tabular}

${ }^{a}$ Three different fluorescent patterns were observed (Figure 3): punctuate, diffuse and mixed.

${ }^{\mathrm{b}}$ The accuracy of the cell sorting process was assessed for the $100 \mathrm{kPa}$ and $500 \mathrm{kPa}$ sample.

${ }^{\mathrm{c}}$ Total number of cells counted. 
As mentioned above, the punctuate distribution of FITC-dextran is assumed to be caused by endocytic uptake. Figure 4 verifies this assumption by assessing the co-localization of FITCdextran with LysoTracker ${ }^{\circledR}$, which stains acidic organelles such as lysosomes. After endocytic uptake, the cargo is transported to endosomes and subsequently lysosomes. Therefore, co-localization of cargo with LysoTracker ${ }^{\circledR}$ indicates endocytic uptake. For cells both untreated and treated with ultrasound, the fluorescence of FITC-dextran co-localizes with LysoTracker®, as indicated by the arrows in Figure 4.
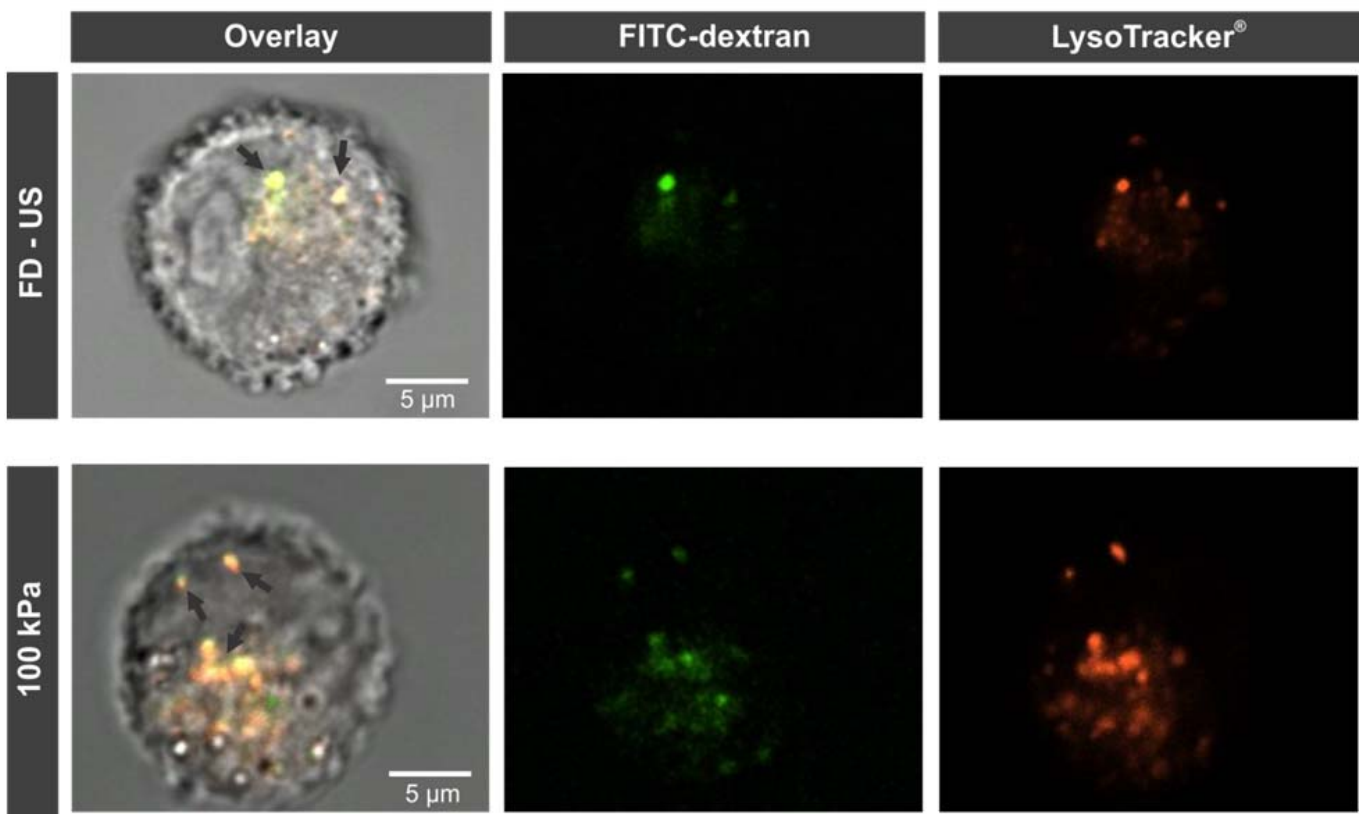

Figure 4. Co-localization of FITC-dextran with LysoTracker ${ }^{\circledR}$. Confocal microscopy images showing the intracellular localization of FITC-dextran (green) and lysosomes stained by LysoTracker®(orange). Co-localization (indicated by the black arrows) confirms that the punctuate distribution of FITC-dextran is due to endocytic uptake, for cells both without (top row) and with (bottom row) ultrasound treatment.

\subsection{Real-time confocal microscopy during ultrasound radiation}

By fluorescently labeling the microbubbles and the cell membrane in green and red, respectively, we were able to visualize microbubble-cell interactions with real-time confocal microscopy. In addition, propidium iodide (PI) was added to the extracellular medium. PI is a cell-impermeable dye, excluded from cells with intact cell membranes. Upon membrane disruption, PI diffuses inwards the cell, where it binds to nucleic acids resulting in an enhanced fluorescence. Therefore, PI is an ideal marker for pore formation. Due to the design of the swept field confocal unit and the broad PI spectra, PI was detected as green fluorescence. The real-time recordings revealed that two main microbubble-cell interactions occurred: (i) microbubbles deforming the cell membrane and (ii) microbubbles propelled towards the cell membrane leading to pore formation. Infrequent interactions $(<5 \%)$ included microbubbles seeming to obstruct pores and microbubbles affecting several cells simultaneously. The latter was more frequently observed at higher pressures and was a violent phenomenon, often leading to cell destruction and cell detachment. An example of membrane deformation by microbubbles is illustrated by a time series of confocal images in Figure 5A (see also Supplementary Video 1). Upon ultrasound exposure, a large microbubble cluster 
(indicated by the circle) moved into the field of view. Afterwards, the cluster started pushing against the cell membrane. Frame 5 shows coalescence of the cluster into one large bubble, which continued to deform the cell membrane. In the last frame, ultrasound is turned off. A clear indentation of the cell membrane was evident, when comparing with the cell boundary contour before ultrasound exposure (marked in all frames with the dotted line). This indentation was accompanied by rearrangement of lipids of the cell membrane, indicated by the red fluorescent rim at the cell border (marked with arrowheads).

A
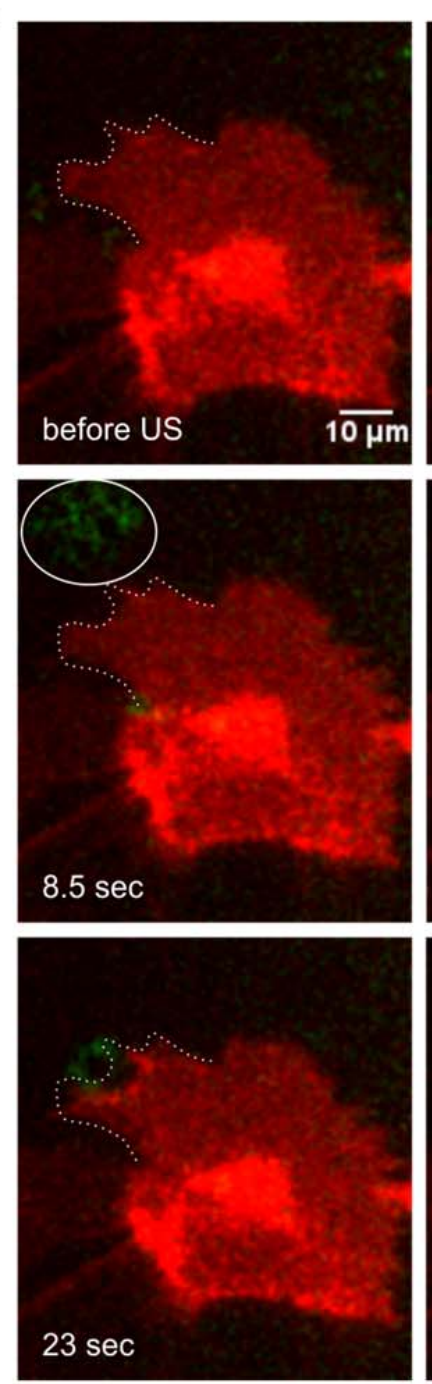

B

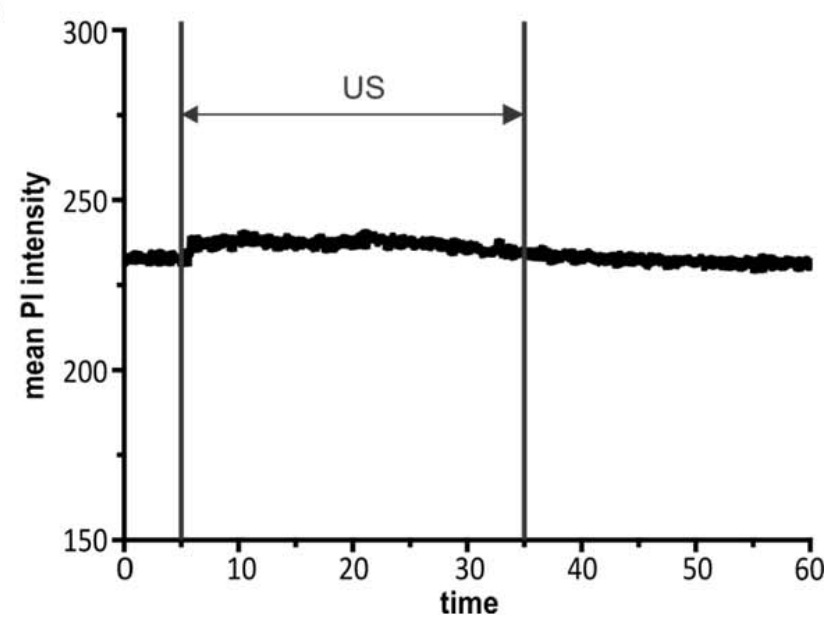

Figure 5. Membrane deformation. Cells were exposed to ultrasound pulses with a center frequency of $1 \mathrm{MHz}$, an acoustic pressure of $200 \mathrm{kPa}$, a pulse length of 2000 cycles, a pulse repetition frequency of $125 \mathrm{~Hz}$ and a total duration of 30 seconds. Acquisition of real-time recordings started $5 \mathrm{sec}$ before the onset of ultrasound exposure. The total duration of the recording was $1 \mathrm{~min}$ and the acquisition rate was 15.61 fps. (A) Time series of confocal images, with the cell membrane and microbubbles labeled in red and green, respectively. The microbubbles (indicated by the circle) clearly deformed the cell membrane during ultrasound exposure, leading to membrane indentation. The pre-exposure cell boundary is marked with the dotted line. This indentation led to an increased density of membrane lipids, indicated by the red fluorescent rim (arrowheads). However, no PI influx was observed. The complete recording is provided in Supplementary Video 1. (B) The mean PI intensity over time, determined by Image $\mathrm{J}$ analysis, confirmed that the cell membrane was not disrupted. 
Nevertheless, no influx of PI was observed, indicating that the membrane was not disrupted. Indeed, image analysis demonstrated there was no increase in mean PI intensity intracellular (Figure 5B). Note that this interaction was provoked not only by microbubble clusters, but also by single microbubbles. Figure $6 \mathrm{~A}$ depicts an example of the second observed interaction (see also Supplementary Video 2). A microbubble (indicated by the full circle) was propelled towards the cell at high velocity. The microbubble trajectory is marked with an arrow. Its impact on the cell membrane created a pore (dotted circle), evidenced by the subsequent influx of PI. It even seems as if the microbubble was able to break through the cell membrane, entering the cytoplasm. Since recordings were acquired with a confocal microscope, a very thin slice of the cell is imaged. Consequently, the microbubble was not located on top or below the cell, but in the cytoplasm. The mean intracellular PI intensity over time is presented in Figure 6B, confirming the observed PI influx. Even when ultrasound was turned off, the mean intensity continued to increase. This might be caused by PI further diffusing into the nucleus, leading to a brighter signal due to the high concentration of nucleic acids. This increased mean intensity might be also due to incomplete pore closure. Pores were reported to be resealed within seconds to minutes [24], while this recording stopped 25 sec after finishing ultrasound exposure.

A
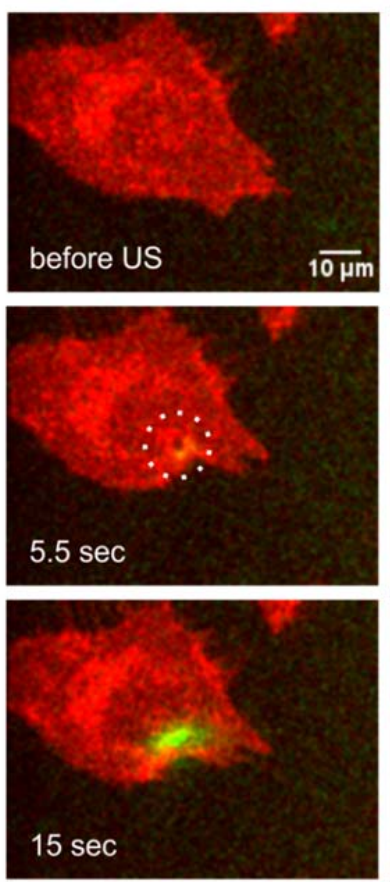

B

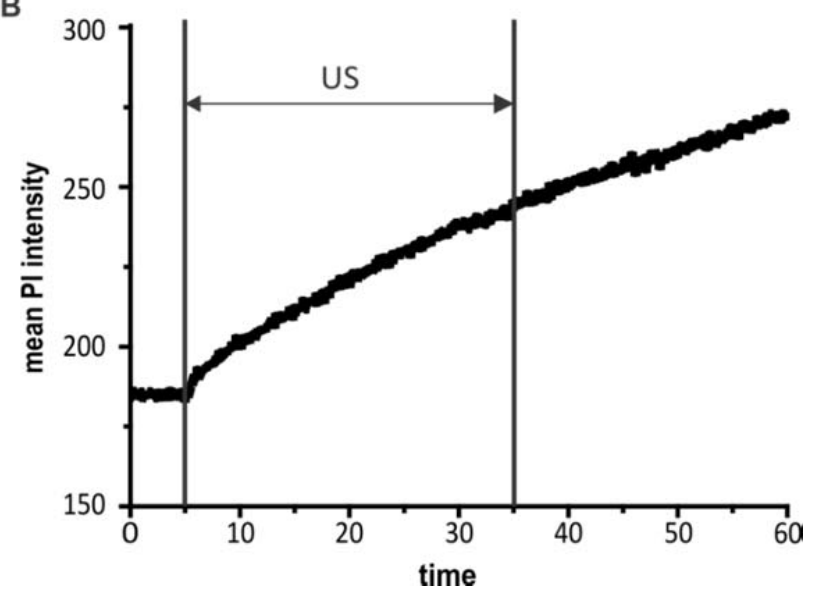

Figure 6. Propelling microbubble causing pore formation. Ultrasound and acquisition settings were the same as for Figure 5. (A) Time series of confocal images, with the cell membrane and microbubbles labeled in red and green, respectively. When ultrasound was turned on, the microbubble (full circle) was propelled towards the cell at high velocity (bubble trajectory indicated by the arrow). Upon collision with the cell membrane, a pore (dotted circle) is created. Consequently, PI diffused inwards the cell. The microbubble even seemed to be pushed through the cell membrane, ending up in the cytoplasm. The complete recording is provided in Supplementary Video 2. (B) The mean PI intensity over time demonstrated the PI influx. This increase in intensity continued after ultrasound exposure due to (i) diffusion into the nucleus leading to a brighter signal or (ii) incomplete pore closure. 
Microbubble behavior depends on the ultrasound settings used. Therefore, recordings were acquired at acoustic pressures ranging from 100 to $500 \mathrm{kPa}$. Cells showing microbubble-cell interactions were counted and classified into the two interactions described above. The infrequent interactions $(<5 \%)$ mentioned above were excluded from this analysis. The results are presented in Figure 7. At each acoustic pressure, both interactions were observed. Membrane deformation was predominant at low acoustic pressure, while its frequency decreased with higher acoustic pressure. The opposite trend is observed for pore formation. The higher the acoustic pressure, the higher the percentage of cells having membrane pores.

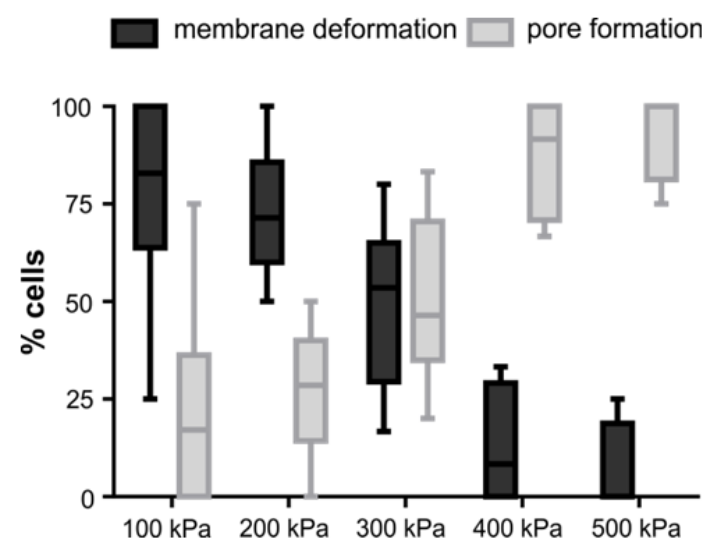

Figure 7. Microbubble-cell interactions at different acoustic pressures. Membrane deformation (illustrated in Figure 5) predominated at low acoustic pressures, while pore formation (illustrated in Figure 6) was more abundant at higher acoustic pressures. Each box plots the minmax range observed over multiple recordings, including 20 to 53 counted cells per acoustic pressure.

\section{DisCUSSION}

\subsection{Dependence of uptake route on acoustic pressure and molecule size}

In literature, contradictory findings have been reported on the involvement of endocytosis in ultrasound mediated delivery. We reasoned that the different ultrasound settings used in these studies may be the cause of this inconsistency. Therefore, we evaluated the uptake mechanisms at a range of acoustic pressures (100-500 kPa). Other acoustic parameters such as center frequency, pulse repetition frequency, pulse length and total exposure time were fixed. Our results demonstrated that the mechanism of drug uptake is indeed dependent on the acoustic pressure applied. Firstly, the uptake of FITC-dextrans at different acoustic pressures was analyzed by flow cytometry. Remarkably, when exposing cells to ultrasound, a low and a high uptake subpopulation arose. Guzmán et al. also reported this heterogeneity in uptake [25]. They suggested this was due to a non-uniform exposure of cells to cavitation. However, this implies that cells would show a whole distribution of intensities of uptake and not two distinct subpopulations. Note that the scale of FITC-intensity in the flow cytometry plots is logarithmic, indicating a large difference in intensity between the subpopulations. In contrast to Guzmán's assumption, our confocal images after cell sorting showed that the subpopulations represented different uptake mechanisms. The low intensity subpopulation showed a punctuate fluorescent pattern, with the dextrans pocketed in endocytic vesicles. The high intensity population represented uptake via pores, resulting in a diffuse fluorescent pattern. Moreover, when increasing the acoustic pressure, the distribution of cells shifted to the high uptake population. Indeed, acoustic pressure was shown to correlate with the size and the number of pores [10]. In conclusion, at low pressures endocytic uptake is promoted, while at higher pressure the fraction of cells in the pore formation population increases. Favoring one of both uptake mechanism by adjusting the acoustic pressure may be exploited for drug delivery. For example, nanomedicines rely on endocytosis to be internalized, as they are too large to be taken up by passive diffusion [26]. These nanomedicines may benefit from an 
enhanced endocytic uptake by exposing cells to low acoustic pressures, while maintaining high viability. However, in some cases the endolysosomal pathway following endocytosis should be avoided. For example, in gene delivery, nucleic acids are degraded in the lysosomes before reaching their target site [27]. In this application, pore formation with direct cytoplasmic entry is desirable. This can be stimulated by using higher acoustic pressures, although a higher toxicity must be considered.

Besides acoustic pressure, molecule size is described in literature to influence the route of internalization as well. Meijering et al. [14] demonstrated that both pore formation and endocytosis were involved in the uptake of dextrans, in agreement with our results. Furthermore, they found a greater contribution of endocytosis for larger molecules. Therefore, we evaluated the uptake of a low and a high molecular weight FITC-dextran, $4 \mathrm{kDa}$ and 2 MDa respectively. Regarding the low intensity population, i.e. the endocytic population, no noteworthy differences were observed between the two molecule sizes. With respect to the pore formation population, higher percentages of positive cells as well as MFIs were obtained for $4 \mathrm{kDa}$ FITC-dextran. Therefore, the contribution of pore formation in the uptake of small dextrans is higher compared to larger dextrans, especially at higher pressures, confirming Meijering's results. Since $4 \mathrm{kDa}$ dextrans are smaller, they can access a larger range of pores sizes. Moreover, they have a higher diffusivity, leading to a more efficient diffusion through pores. This finding is confirmed by several other studies, reporting a correlation between molecule size and delivery efficiency $[15,19]$. It must be noted though that for both dextrans, the same weight concentration instead of the same molar concentration was used, due to the detection limit of the flow cytometer. Consequently, a higher number of $4 \mathrm{kDa}$ dextran molecules was presented to the cells. Nevertheless, one $4 \mathrm{kDa}$ FITC-dextran molecule contains less fluorescent labels than one $2 \mathrm{MDa}$ molecule. Therefore, it remains difficult to directly compare these two different MW dextrans, especially in terms of MFI.

To study endocytosis, chemical endocytosis inhibitors as well as more general treatments to block endocytosis, e.g. lowering the temperature to $4^{\circ} \mathrm{C}$ or ATP depletion, are frequently used. These tools were also applied in studies to investigate the role of endocytosis in ultrasound mediated delivery [14, 15, 19, 20]. Cells display various pathways of endocytic internalization, with macropinocytosis, clathrin-mediated and caveolae-mediated endocytosis being the best studied ones [27]. Chemical inhibitors are used to block one of these pathways. However, they lack specificity. Moreover, inhibiting one pathway may upregulate other compensatory pathways [28]. Furthermore, they are toxic and may interfere with ultrasound mediated effects. For example, actin-disrupting agents, e.g. cytochalasin, may change the cytomechanical vulnerability to physical stimuli such as ultrasound [29]. Yu and colleagues indeed showed that sonoporation not only affects the cell membrane, but also causes reorganization of the cytoskeleton [30]. Therefore, results obtained with chemical inhibitors should be carefully interpreted. Incubation of cells at $4^{\circ} \mathrm{C}$ is a method to generally block endocytosis. However, this also influences the stiffness of the lipid microbubble shell [31], thereby changing the ultrasound-responsiveness and behavior of the microbubbles [32]. Moreover, cell membrane fluidity is affected by temperature and this may again alter the vulnerability of cells to sonoporation [33]. A lower temperature as well as ATP depletion may hinder pore repair, since this is an energy-dependent process [19]. To minimize manipulation of cellular processes, we preferred to confirm endocytic uptake by co-localization with the general marker LysoTracker ${ }^{\circledR}$, which stains lysosomes. Lysosomes are part of the intracellular route following endocytosis, mostly independent of which specific endocytic pathway was addressed. Co-localization of FITC-dextran and LysoTracker® was observed for cells both untreated and treated with ultrasound. This confirmed that the punctuate pattern of FITC-dextran indeed represented endocytic uptake. 


\subsection{Link between microbubble-cell interactions and uptake mechanism}

The dependence of the uptake route on acoustic pressure may be explained by microbubble behavior. When varying the acoustic pressure, microbubbles will behave differently. Besides pressure, other acoustic (e.g. center frequency, pulse repetition frequency and pulse length) or microbubble related (e.g. size and shell type) parameters will influence microbubble behavior as well [34]. Furthermore, microbubble concentration influences microbubble-cell distance and chance of interaction [35]. The complex interplay between all these parameters will determine how microbubbles affect cells. Consequently, different microbubble-cell interactions may induce different routes of drug internalization. With high-speed bright-field microscopy, the effects of microbubbles on cells have been studied before [36-38]. However, this technique does not provide information on drug uptake. Fluorescence microscopy can be used to monitor the uptake of fluorescent probes, serving as model drugs [10, 39]. Besides using fluorescent probes, we fluorescently labeled microbubbles as well as cells. In this way, microbubble-cell interactions may be linked to mechanisms of drug uptake. Moreover, with our swept field confocal setup, a fast and high contrast rendering of the microbubble-cell interactions was obtained. Furthermore, most studies use single shot ultrasound and manipulate experimental conditions to have single bubbles, which is often not the case in drug delivery studies. Therefore, we used in all experiments similar conditions to be able to relate microscopic observations to uptake results.

The real-time recordings revealed that low acoustic pressures mainly caused membrane deformation without disrupting the membrane. Radiation forces are playing a major role in this phenomenon. The primary radiation force drives microbubbles in the direction of the ultrasound beam [40]. When encountering a cell, this radiation force compresses the microbubble onto the cell membrane, resulting in membrane indentation without disruption. Fan et al. [41] and Zhou et al. [42] reported similar observations. The secondary radiation force causes microbubble clustering [43], as illustrated in Figure 5. In addition to radiation force, microstreamings may also contribute to the observed effects [44]. The microbubbles are cavitating stably, since they exist throughout the ultrasound exposure. This stable cavitation creates microstreamings in the surrounding fluid, exerting shear stresses on the cell membrane, which may aid in membrane deformation. We hypothesize that this membrane deformation stimulates endocytosis, since this is the main observed uptake route at low acoustic pressure. Generally, it is described in literature that alterations in membrane tension, induced by membrane deformation, are accompanied by a reorganization of the underlying cytoskeleton. Subsequently, mechanosensors, e.g. integrins, are activated and regulate endocytosis and exocytosis processes to normalize membrane tension [45]. In case of ultrasound exposed cells, remodeling of the cytoskeleton has been shown as well [30,46]. Moreover, mechanotransduction pathways were reported to be activated upon ultrasound exposure [47, 48]. Besides this cytoskeletal mediated hypothesis, two other mechanisms by which endocytosis may be upregulated are postulated in literature. Both regard endocytosis as a consequence of pore formation. Firstly, the rise in intracellular calcium levels, provoked by membrane poration, is proposed to stimulate endocytosis [49]. The second hypothesis originates from pore repair. In mechanically injured cells, small lesions were found to be resealed by endocytosis [50]. The same processes may apply for ultrasound exposed cells, since they are also wounded in a mechanical way [8]. However, our confocal recordings rather support the cytoskeletal stimulated endocytosis assumption.

Besides membrane deformation, a second microbubble-cell interaction was observed, i.e. propelling microbubbles causing membrane disruption. This interaction occurred more frequently when acoustic pressure was increased. Correspondingly, the fraction of cells in the 
pore formation population in flow cytometry experiments increased. At higher pressures, the generated radiation force is stronger, leading to a more violent microbubble translation. When impinging on the cell, the membrane is disrupted, proven by the influx of PI. As depicted in Figure 6, the microbubble may even break through the cell membrane, entering the cell cytoplasm. In general, microbubbles are believed to act in the cell surroundings. However, Delalande et al. [51] also demonstrated microbubbles entering cells during ultrasound radation by bright field imaging. Due to the confocality of our recordings, the intracellular localization of the bubbles is confirmed. This implies that loading the microbubbles with drugs may be beneficial to achieve high delivery rates. It would bring the drug exactly to the site of pore formation, or even directly in the cytoplasm.

\section{CONCLUSION}

Our findings indicate that acoustic pressure influences the uptake mechanism addressed in ultrasound mediated delivery. Real-time imaging during ultrasound exposure enabled us to record different microbubble-cell interactions when varying acoustic pressure. The limitation of our study is that only acoustic pressure was varied, while other acoustic and microbubble related parameters influence these interactions as well. However, acoustic pressure is a major determinant of microbubble behavior. As a consequence, we were able to direct cellular uptake towards endocytosis or direct cytoplasmic entry via pores, solely by changing acoustic pressure. Low acoustic pressure enhanced uptake by mainly stimulating endocytosis. Realtime recordings revealed that at these pressures gentle membrane deformation occurred. In literature, membrane deformation is linked to upregulated endocytosis. In contrast, high acoustic pressures lead to uptake via membrane pores. The primary radiation force propelled microbubbles towards cells at high velocity. Upon collision, pores were created in the cell membrane. When designing drug delivery experiments, these findings may be considered in order to select optimal ultrasound settings. In this way, drugs are delivered at the right site, which may boost therapeutic efficiency.

\section{Acknowledgements}

Ine De Cock is a doctoral fellow of the Institute for the Promotion of Innovation through Science and Technology in Flanders, Belgium (IWT-Vlaanderen). Ine Lentacker is a postdoctoral fellow of the Research Foundation Flanders, Belgium (FWO-Vlaanderen). The support of both these institutions is gratefully acknowledged. We would also like to thank Robert Beurskens and Michiel Manten for their technical support. Bert Devriendt is thanked for the assistance in the FACS sorting experiments.

\section{Reference list}

1. K. Tachibana, S. Tachibana, The Use of Ultrasound for Drug Delivery, Echocardiography 18 (4) (2001) 323-328.

2. $\quad$ E.L. Yuh, S.G. Shulman, S.A. Mehta, J. Xie, L. Chen, V. Frenkel, M.D. Bednarski, K.P.C. Li, Delivery of Systemic Chemotherapeutic Agent to Tumors by Using Focused Ultrasound: Study in a Murine Model, Radiology 234 (2) (2005) 431-437.

3. R. Bekeredjian, S. Chen, P.A. Grayburn, R.V. Shohet, Augmentation of cardiac protein delivery using ultrasound targeted microbubble destruction, Ultrasound Med. Biol. 200531 (5) (2005) 687-691.

4. M. De Temmerman, H. Dewitte, R.E. Vandenbroucke, B. Lucas, C. Libert, J. Demeester, S.C. De Smedt, I. Lentacker, J. Rejman, mRNA-lipoplex loaded 
microbubble contrast agents for ultrasound-assisted transfection of dendritic cells, Biomaterials 32 (34) (2011) 9128-9135.

5. S. Sirsi, M.A. Borden, State-of-the-art materials for ultrasound-triggered drug delivery, Adv. Drug Deliver. Rev. 72 (2014) 3-14.

6. E. Stride, Physical Principles of Microbubbles for Ultrasound Imaging and Therapy, Cerebrovasc. Dis. 27 (Suppl 2) (2009) 1-13.

7. $\quad$ E. Quaia, Microbubble ultrasound contrast agents: an update, Eur. Radiol. 17 (2007) 1998-2005.

8. I. Lentacker, I. De Cock, R. Deckers, S.C. De Smedt, C.T.W. Moonen, Understanding ultrasound induced sonoporation: Definitions and underlying mechanisms, Adv. Drug Deliver. Rev. 72 (2014) 49-64.

9. K. Tachibana, T. Uchida, N. Yamashita, K. Tamura, Induction of cell-membrane porosity by ultrasound, Lancet 353 (9162) (1999) 1409.

10. Z. Fan, H. Liu, M. Mayer, C.X. Deng, Spatiotemporally controlled single cell sonoporation, P. Natl. Acad. Sci. USA 109 (41) (2012) 16486-16491.

11. N. Kudo, K. Okada, K. Yamamoto, Sonoporation by single-shot pulsed ultrasound with microbubbles adjacent to cells, Biophys. J. 96 (12) (2009) 4866-4876.

12. A. van Wamel, K. Kooiman, M. Harteveld, M. Emmer, F.J. ten Cate, M. Versluis, N. de Jong, Vibrating microbubbles poking individual cells: Drug transfer into cells via sonoporation, J. Control. Release 112 (2) (2006) 149-155.

13. H.R. Guzman, D.X. Nguyen, S. Khan, M.R. Prausnitz, Ultrasound-mediated disrutpion of cell membranes. I. Quantification of molecular uptake and cell viability, J. Acoust. Soc. Am. 110 (1) (2001) 588-596.

14. B.D.M. Meijering, L.J.M. Juffermans, A. van Wamel, R.H. Henning, I.S. Zuhorn, M. Emmer, A.M.G. Versteilen, W.J. Paulus, W.H. van Gilst, K. Kooiman, N. de Jong, R.J.P. Muster, L.E. Deelman, O. Kamp, Ultrasound and microbubble-targeted delivery of macromolecules is regulated by induction of endocytosis and pore formation, Circ. Res. 104 (5) (2009) 679-687.

15. M. Afadzi, S.P. Strand, E.A. Nilssen, S.E. Måsøy, T.F. Johansen, R. Hansen, B.A. Angelsen, C. de L. Davies, Mechanisms of the ultrasound-mediated intracellular delivery of liposomes and dextrans, IEEE T. Ultrason. Ferr. 60 (1) (2013) 21-33.

16. J. Hauser, M. Ellisman, H. Steinau, E. Stefan, M. Dudda, M. Hauser, Ultrasound enhanced endocytotic activity of human fibroblasts, Ultrasound Med. Biol. 35 (12) (2009) 2084-2092.

17. V. Lionetti, A. Fittipaldi, S. Agostini, M. Giacca, F.A. Recchia, E. Picano, Enhanced caveolae-mediated endocytosis by diagnostic ultrasound in vitro, Ultrasound Med. Biol. 35 (1) (2009) 136-143.

18. D.M. Paula, V.B. Valero-Lapchik, E.J. Paredes-Gamero, S.W. Han, Therapeutic ultrasound promotes plasmid DNA uptake by clathrin-mediated endocytosis, J. Gene Med. 13 (7-8) (2011) 392-401.

19. R. Schlicher, H. Radhakrishna, T.P. Tolentino, R.P. Apkarian, V. Zarnitsyn, M.R. Prausnitz, Mechanism of intracellular delivery by acoustic cavitation, Ultrasound Med. Biol. 32 (6) (2006) 915-924.

20. I. Lentacker, N. Wang, R.E. Vandenbroucke, J. Demeester, S.C. De Smedt, N.N. Sanders, Ultrasound exposure of lipoplex loaded microbubbles facilitates direct cytoplasmic entry of lipoplexes, Mol. Pharmaceutics 6 (2) (2009) 457-467.

21. P.H.A. Quax, E.J.D. Vanmuijen, L.R. Weeningverhoeff, K. Lund, D.J. Dano, J.H. Ruiter, J. Verheijen, Metastatic behavior of human melanoma cell lines in nude mice correlates with urokinase-type plasminogen activator, its type-1 inhibitor, and urokinase-mediated matrix degradation, J. Cell Biol. 115 (1) (1991) 191-9. 
22. K. Braeckmans, L. Peeters, N.N. Sanders, S.C. De Smedt, J. Demeester, Threedimensional fluorescence recovery after photobleaching with the confocal scanning laser microscope, Biophys. J. 85 (4) (2003) 2240-2252.

23. C. Plank, B. Oberhauser, K. Mechtler, C. Koch, E. Wagner, The influence of endosome-disruptive peptides on gene transfer using synthetic virus-like gene transfer systems, J. Biol. Chem. 269 (17) (194) 12918-12924.

24. Y. Hu, J. Wan, A. Yu, Membrane Perforation and Recovery Dynamics in Microbubble-Mediated Sonoporation, Ultrasound Med. Biol. 39 (12) (2013) 23932405.

25. H.R. Guzman, D.X. Nguyen, S. Khan, M.R. Prausnitz, Ultrasound-mediated disruption of cell membranes.II Heterogeneous effects on cells, J. Acoust. Soc. Am., 110 (1) (2001) 597-606.

26. R. Duncan, S.C.W. Richardson, Endocytosis and intracellular trafficking as gateways for nanomedicine delivery: opportunities and challenges, Mol. Pharmaceutics 9 (9) (2012) 2380-2402.

27. D. Vercauteren, J. Rejman, T.F. Martens, J. Demeester, S.C. De Smedt, K. Braeckmans, On the cellular processing of non-viral nanomedicines for nucleic acid delivery: mechanisms and methods, J. Control. Release 161 (2) (2012) 566-581.

28. D. Vercauteren, R.E. Vandenbroucke, A.T. Jones, J. Rejman, J. Demeester, S.C. Desmedt, N.N. Sanders, K. Braeckmans, The Use of Inhibitors to Study Endocytic Pathways of Gene Carriers: Optimization and Pitfalls, Mol. Ther. 18 (3) (2009) 561569.

29. Y. Ujihara, M. Nakamura, H. Miyazaki, S. Wada, Contribution of actin filaments to the global compressive properties of fibroblasts, J. Mech. Behav. Biomed. Mater. 14 (2012) 192-198.

30. X. Chen, R.S. Leow, Y. Hu, J.M. Wan, A.C. Yu, Single-site sonoporation disrupts actin cytoskeleton organization, J. R. Soc. 11 (95) (2014) 20140071.

31. C.A. Grant, J.E. McKendry, S.D. Evans, Temperature dependent stiffness and viscoelastic behaviour of lipid coated microbubbles using atomic force microscopy, Soft Matter 8 (5) (2012) 1321-1326.

32. H. Mulvana, E. Stride, M. Tang, J.V. Hajnal, R. Eckersley, Temperature-dependent differences in the nonlinear acoustic behavior of ultrasound contrast agents revealed by high-speed imaging and bulk acoustics, Ultrasound Med. Biol. 37 (9) (2011) 15091517.

33. T. Nozaki, R. Ogawa, L.B.Jr. Feril, G. Kagiya, H. Use, T. Kondo, Enhancement of ultrasound-mediated gene transfection by membrane modification, J. Gene Med. 5 (12) (2003) 1046-1055.

34. V. Sboros, Response of contrast agents to ultrasound, Adv. Drug Deliver. Rev. 60 (10) (2008) 1117-1136.

35. H.R. Guzmán, A.J. McNamara, D.X. Nguyen, M.R. Prausnitz, Bioeffects caused by changes in acoustic cavitation bubble density and cell concentration: a unified explanation based on cell-to-bubble ratio and blast radius, Ultrasound Med. Biol. 29 (8) (2003) 1211-1222.

36. A. van Wamel, A. Bouakaz, M. Versluis, N. de Jong, Micromanipulation of endothelial cells: ultrasound-microbubble-cell interaction, Ultrasound Med. Biol. 30 (9) (2004) 1255-1258.

37. D. Ohl, M. Arora, R. Ikink, N. de Jong, M. Versluis, M. Delius, D. Lohse, Sonoporation from jetting cavitation bubbles, Biophys. J. 91 (11) (2006) 4285-4295. 
38. P. Prentice, A. Cuschieri, K. Dholakia, M. Prausnitz, P. Campbell, Membrane disrutpion by optically controlled microbubble cavitation, Nat. Phys. 1 (2005) 107110.

39. M. Derieppe, A. Yudina, M. Lepetit-Coiffé, B.D. de Senneville, C. Bos, C. Moonen, Real-time assesment of ultrasound-mediated drug delivery using fibered confocal fluorescence microscopy, Mol. Imaging Biol. 15 (1) (2012) 3-11.

40. M. Postema, A. van Wamel, C.T. Lancée, N. de Jong, Ultrasound-induced encapsulated microbubble phenomena, Ultrasound Med. Biol. 30 (6) (2004) 827-840.

41. Z. Fan, D. Chen, C.X. Deng, Characterization of the Dynamic Activities of a Population of Microbubbles Driven by Pulsed Ultrasound Exposure in Sonoporation, Ultrasound Med. Biol. 40 (6) (2014) 1260-1272.

42. Y. Zhou, K. Yang, J. Ciu, J.Y. Ye, C.X. Deng, Controlled permeation of cell membrane by single bubble acoustic cavitation, J. Control. Release 157 (1) (2012) 103-111.

43. S. Kotopoulis, M. Postema, Microfoam formation in a capillary, Ultrasonics 50 (2) (2010) 260-268.

44. S. Nejad, S.H.R. Hosseini, H. Akiyama, K. Tachibana, Optical observation of cell sonoporation with low intensity ultrasound, Biochem. Bioph. Res. Co. 413 (2) (2011) 218-223.

45. G. Apodaca, Modulation of membrane traffic by mechanical stimuli, Am. J. Physiol.Renal 282 (2) (2002) 90.

46. Z. Fan, Y. Sun, C. Di, D. Tay, W. Chen, C.X. Deng, Acoustic tweezing cytometry for live-cell subcellular modulation of intracellular cytoskeleton contractility, Sci. Rep. 3 (2013) 2176.

47. N.P. Whitney, A.C. Lamb, T.M. Louw, A. Subramanian, Integrin-mediated mechanotransduction pathway of low-intensity continuous ultrasound in human chondrocytes, Ultrasound Med. Biol. 38 (10) (2012) 1734-1743.

48. T.M. Louw, G. Budhiraja, H.J. Viljoen, A. Subramanian, Mechanotransduction of Ultrasound is Frequency Dependent Below the Cavitation Threshold, Ultrasound in Med. Biol. 39 (7) (2013) 1303-1319.

49. L.J.M. Juffermans, O. Kamp, P. Dijkmans, C. Visser, R. Musters, Low-intensity ultrasound-exposed microbubbles provoke local hyperpolarization of the cell membrane via activation of BKCa channels, Ultrasound Med. Biol. 34 (3) (2008) 502508.

50. A. Draeger, K. Monastyrskaya, E. Babiychuk, Plasma membrane repair and cellular damage control: the annexin survival kit, Biochem. Pharmacol. 81 (6) (2011) 703-712.

51. A. Delalande, S. Kotopoulis, T. Rovers, C. Pichon, M. Postema, Sonoporation at a low mechanical index, Bubble Science, Engineering \& Technology 3 (1) (2001) 3-11. 


\section{Supplementary data}

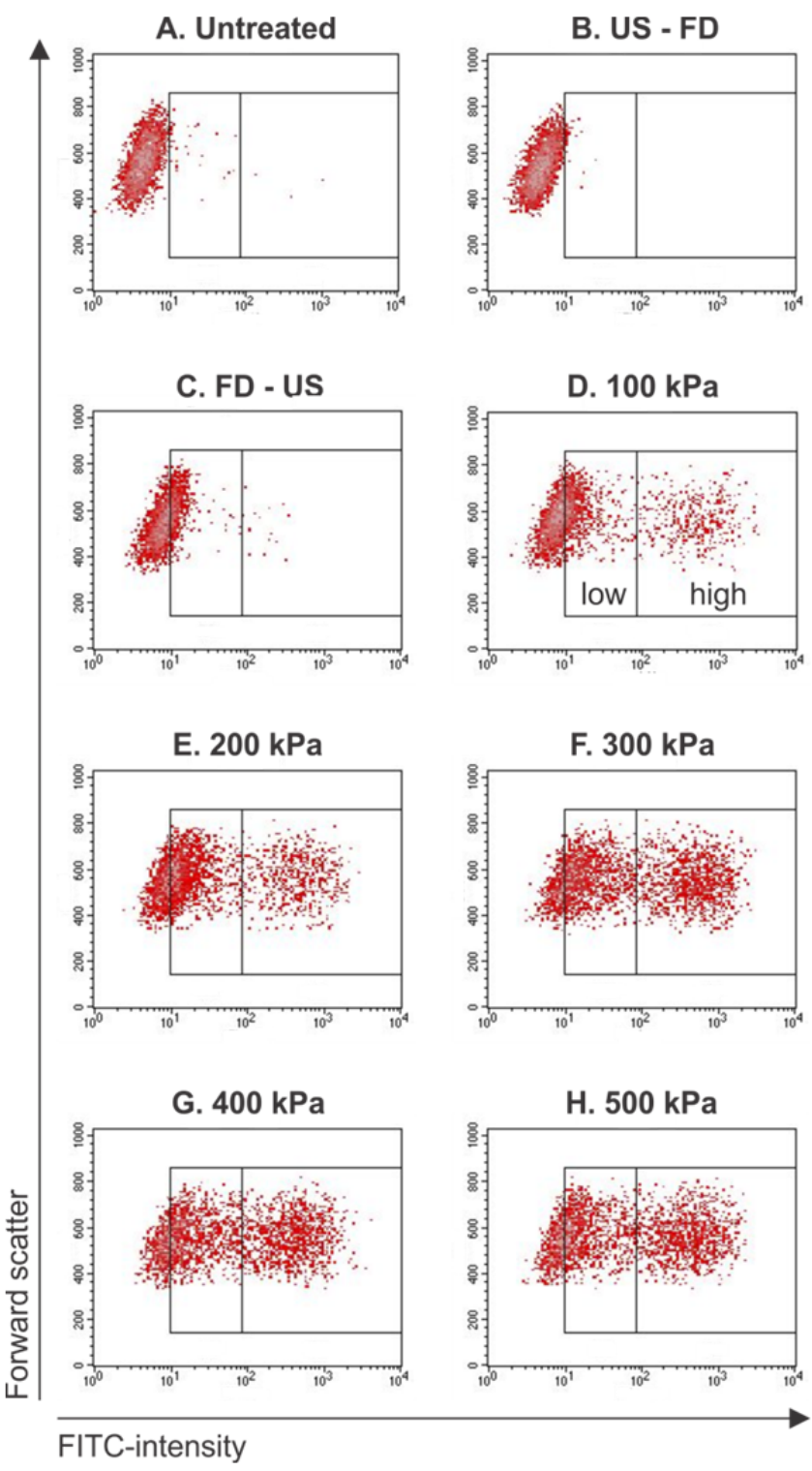

Supplementary Figure 1. Scatterplots (Forward scatter - FITC-fluorescence intensity) of the uptake of 2 MDa FITC-dextran. (A) Cells not treated with FITC-dextran or ultrasound. (B) Sonicated cells, without FITC-dextran incubation. (C) Cells incubated with FITC-dextran without ultrasound exposure. (D)-(H) Cells incubated with FITC-dextran and exposed to ultrasound with increasing acoustic pressure. When exposing cells to ultrasound, two subpopulations arose. These low and high intensity subpopulations were gated as indicated. Similar plots were obtained for 4 kDa FITC-dextran. 

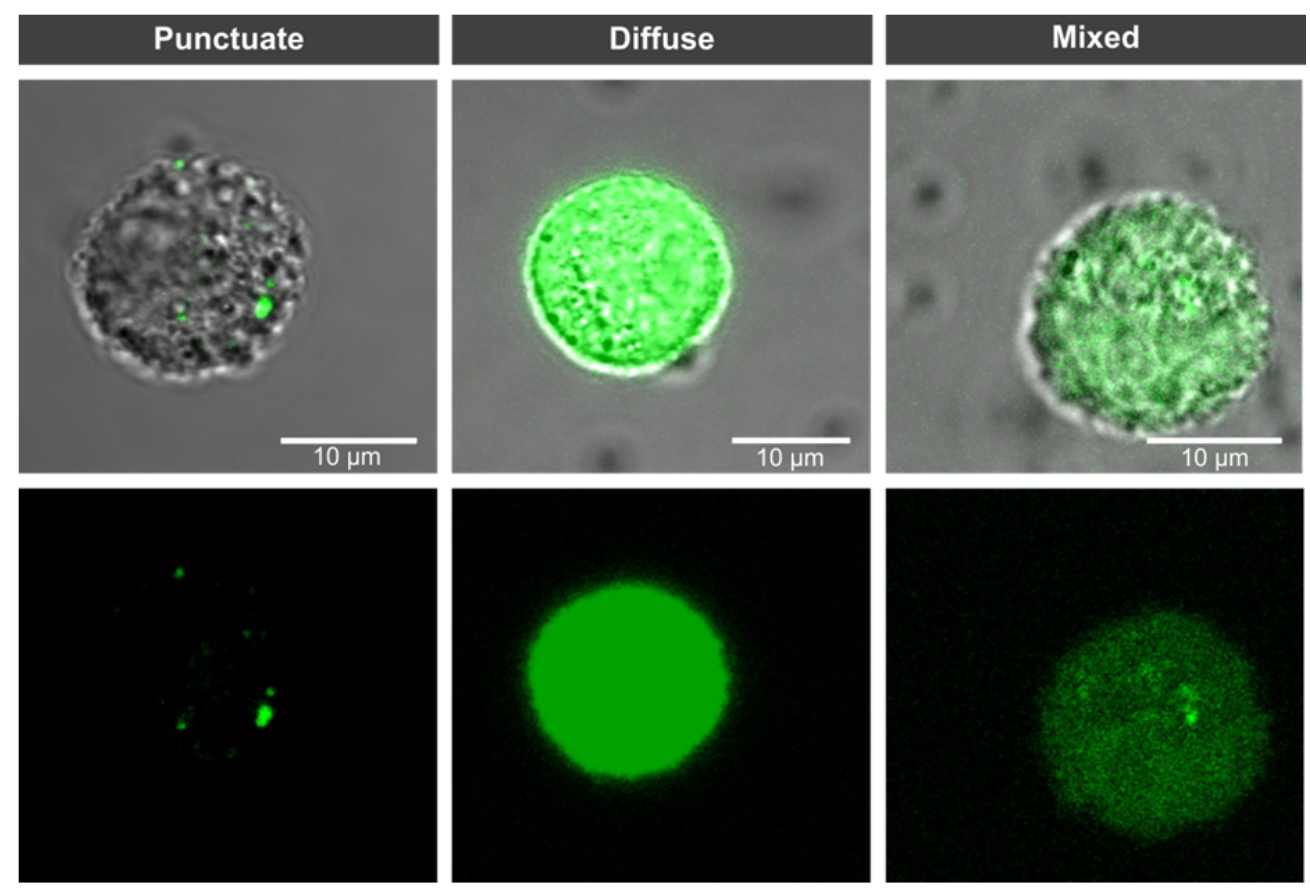

Supplementary Figure 2. Intracellular localization of 4 kDa FITC-dextran. Confocal microscopy images with the top rows depicting an overlay of the transmission and FITCfluorescence image; the bottom rows only show FITC-fluorescence. Similarly to $2 \mathrm{MDa}$ FITC-dextran, three different fluorescent patterns were observed: punctuate, diffuse and mixed. In contrast to $2 \mathrm{MDa}$ FITC-dextran, $4 \mathrm{kDa}$ dextrans also appear in the nucleus, since they are small enough to be transported across the nuclear membrane.

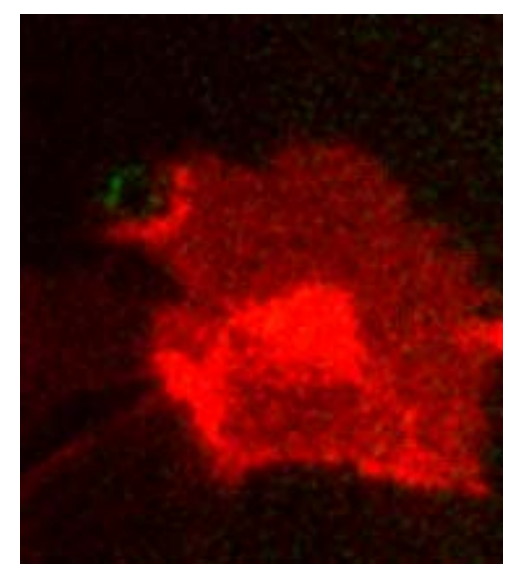

Supplementary Video 1. Microbubble causing membrane deformation.

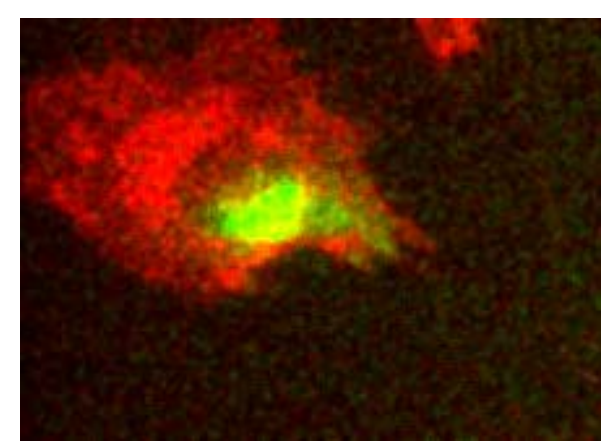

Supplementary Video 2. Propelling microbubble causing pore formation. 